\title{
Perspectives of constant gradient magnetic fields applications in biotechnology
}

\author{
Tamara A. Ignatyeva ${ }^{1}$, Victor N. Voyevodin ${ }^{1}$, Anatoly N. Goltsev ${ }^{2}$, Victoria V. Kiroshka ${ }^{2}$, \\ Alexander M. Bovda ${ }^{1}$, Valery V. Kalynovskii ${ }^{1}$, Alexey N. Velikodny ${ }^{1}$, Peter A. Kutsenko ${ }^{1}$, \\ Vladimir Golub ${ }^{3}$, Yuri Dzhedzheria ${ }^{3}$, Irina Sharai ${ }^{3}$
}

${ }^{1}$ National Scientific Center "Kharkov Institute of Physics and Technology”, 61108 Kharkov, Ukraine

${ }^{2}$ Institute for Problems of Cryobiology and Cryomedicine NASU, 61015 Kharkov, Ukraine

${ }^{3}$ Institute of Magnetism NASU and MESU, $03142 \mathrm{Kiev}$, Ukraine

\section{Email address:}

taignatieva@mail.ru (T. A. Ignatyeva), vvkiroshka@mail.ru (V. V. Kiroshka)

\section{To cite this article:}

Tamara A. Ignatyeva, Victor N. Voyevodin, Anatoly N. Goltsev, Victoria V. Kiroshka, Alexander M. Bovda, Valery V. Kalynovskii, Alexey N. Velikodny, Peter A. Kutsenko, Vladimir Golub, Yuri Dzhedzheria, Irina Sharai. Perspectives of Constant Gradient Magnetic Fields Applications in Biotechnology. American Journal of Bioscience and Bioengineering. Vol. 2, No. 6, 2014, pp. $72-77$. doi: 10.11648/j.bio.20140206.11

\begin{abstract}
Elastic hard magnetic materials based resin-bond magnets with the determined space configuration of the magnetic field required for a three-dimensional cell growth which is essential for the tissue engineering have been produced. Technical tests of the samples as well as the theoretical study of the distribution of stray fields produced by ferromagnetic particles correspondingly distributed in the film have been carried out. In vitro experimental investigations of the gradient magnetic field influence on a cell differentiation on transplanted epithelial-like kidney cells culture of a pig embryo has been carried out. It has been shown that the adhesion, morphology and proliferation rate of the cells is determined not only by the magnetic field value but also by its gradient direction. It has been established that the cell adhesion efficiency is the highest when the magnetic field gradient is directed from the Petri dish bottom to the air-culture medium interface. The obtained results prove the possibility of an implementation of new gradient magnetic fields based methods in biotechnology and in particular in tissue engineering.
\end{abstract}

Keywords: Resin-Bond Magnets, Cell Culture, Magnetic Field, Gradient Magnetic Field, Adhesion, Proliferation

\section{Introduction}

One of the top priorities of the modern biotechnology is the development of tissue engineering to create the biological tissues and organs, which are analogous to the living ones. Nowadays the tissue transplantation science is being the most important field of biology and medicine. The tissue engineering methods are targeted in the long run to solve the organ transplantation issue and the replacement of affected tissues with their in vitro produced equivalents.

Tissues or cells of the biological object can serve as the initial material in this case. The growth of tissues with the desired metabolic and other properties do not just require growth media but also the facilitation of the 3D cell growth processes which are essential for the living biological tissues reproduction. Formation of the tissue 3D structure is one of the key challenges of the tissue engineering. The most widely used 3D tissue growth method nowadays is the creation of matrixes using biocompatible polymer materials. Constant magnetic fields are considered to be used for the control 3D cell culture growth. It has been shown in [1], the cell differentiation process may be controlled by magnetic field using $\mathrm{Fe}_{3} \mathrm{O}_{4}$ nanoparticles based magnetic gel by generation of weightlessness environment.

Here we proposed an alternative approach of magnetic field controlled tissue engineering based on generation of anisotropic energetic space for cell growth using space periodic gradient magnetic fields. The methods for such gradient magnetic fields generation have been developed and investigated [2] based on supposition of possibility to provide a condition for $3 \mathrm{D}$ cell growth $[3,4]$ without an employment of any matrixes or nanoparticles being incorporated into cells 
which may allow producing healthy tissues for the practical purpose in "pure form". The mechanisms of the magnetic field influence are still not clear enough. But our recent experiments have shown the influence of gradient magnetic fields with the defined space configuration on 3D cell growth which could open new ways for tissue engineering.

From the practical point of view resin-bonded magnets with determined magnetic parameters are the most convenient sources of magnetic field because they are compact, can provide desired gradient magnetic field configuration for cells growth and could be used both in vitro and in vivo. Such resin-bonded magnets have been developed, their technical characteristic have been established and test experiments on cell cultures have been conducted.

So far there is no definite conception of biological effects caused by static magnetic fields (SMF) which were observed both in the natural environment and during the studies conducted in vivo and in vitro [5]. However depending on the biological impact of the SMF the following classification of magnetic fields (MF) has been adopted: weak MF $(<1 \mathrm{mT})$, medium MF (from $1 \mathrm{mT}$ to $1 \mathrm{~T}$ ), strong MF (from 1 to $5 \mathrm{~T}$ ) and very strong MT (> 5 T) [6-8]. The geomagnetic field providing guidance for the creatures with a well-developed biogenic magnetite system also belongs to weak MF. Due to the practical incorporation of new medical treatment methods, namely magnetotherapy and hyperthermia, the study of biological effects of external MF of weak and medium range with a certain magnetic field gradient distribution is of great interest today. Strong and very strong SMF, being used during MRI in particular, are able to change orientation of different anisotropic diamagnetic organic molecules, possibly having many negative effects on the organs altogether $[9,10]$. Investigations of weak and medium range MFs biological effects on different biosystems are often questionable and of phenomenological nature, revealing no action mechanisms [9 11]. The methods for the generation of weak magnetic fields of different configuration as well as the investigation of the influence such fields on cells adhesion, morphology and proliferation is presented in this work.

\section{Materials and Methods}

\subsection{Samples Characterization}

Plate-like resin-bonded magnets with a determined distribution of hard magnetic material particles were used in our biological experiments as the external magnetic field source.

Biological experiments have been conducted on transplanted culture of growing in the Sanyo planted culture of SPEV -13-D5-TK cell line (epithelial-like kidney cells of a pig embryo). The growth medium used for all cells was Dulbecco's modification of Eagle's medium (DMEM) supplemented with $10 \%$ (v/v) fetal calf serum (FCS) and penicillin $(100 \mathrm{U} / \mathrm{ml})$ and streptomycin $(100 \mathrm{mg} / \mathrm{ml})(\mathrm{Gibco}$, Life Technologies, Paisley, Scotland, UK). All cells were cultured in $30 \mathrm{~mm}$ Petri dishes (Nunc) with $2 \mathrm{ml}$ medium at 37
${ }^{0} \mathrm{C}$ in $5 \%, \mathrm{CO}_{2}$ (Sanyo). Cells concentration in the $30 \mathrm{~mm}$ diameter Petri dish by the inoculation was $1.9-2.1 \times 10^{5}$ cells/ml.
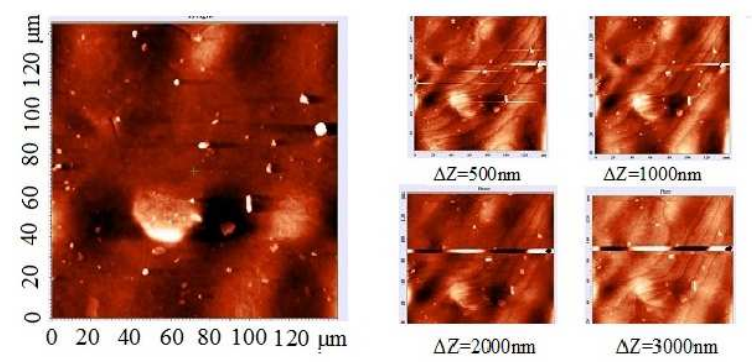

a)
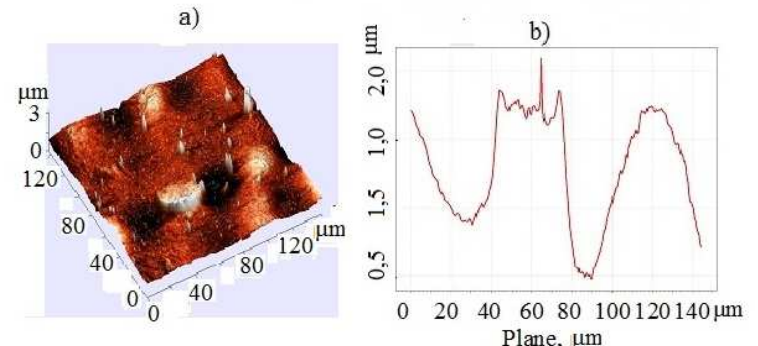

Fig. 1. a) The resin-bonded magnets surface topography, b) $3 d$ image, (c) $M F M$ at different $\Delta z$ distances from the sample surface. $(g$ ) cross-section to the sample surface at $y=60 \mu \mathrm{m}$.

Magnetic force microscopy (MFM SolverPro) was used to study magnetic field distribution over the resin-bonded magnets surface. In order to get MFM magnetic samples images the double pass method [12] has been applied. The magnetic field distribution over the surface at different distances from the plate is shown in Fig. 1.

The comparison of AFM and MFM images has revealed that the magnetic potential distribution is mainly determined by the sample surface geometry. The obtained results are in good correlation with the optical microscopy the soft magnetic substrates which were used during the preparation of resin-bonded magnets (see Fig.2).
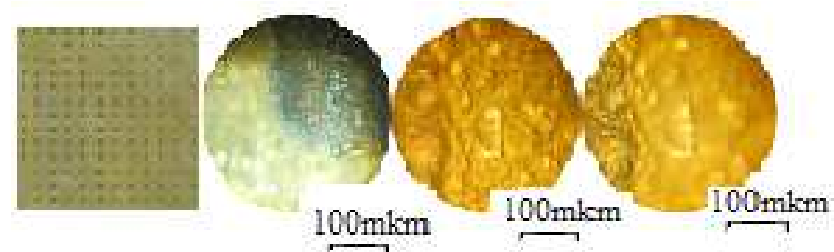

Fig. 2. a) Optical image of the soft magnetic substrate: the distance between the squares centers is $1.65 \mathrm{~mm}$. Squares side is 0.35-0.4 mm. b) the middle of the step is $20 \mu \mathrm{m}$; c) bottom of the step; d) top of the step.

\subsection{Theoretical Calculation of Stray Fields}

The magnetic field distribution has been calculated theoretically using the model structure presented in Fig.3, which is the square array of magnetic rods. The distance between the rods is $a$, their height and diameter are $z_{0}$ i $d$ correspondingly, the rods are magnetized to the saturation magnetization $M_{S}$ along $z$ axis. 


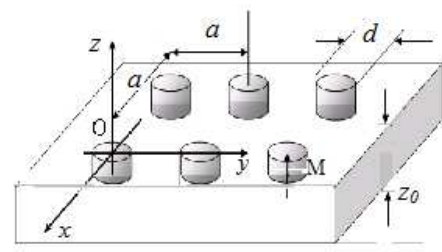

a

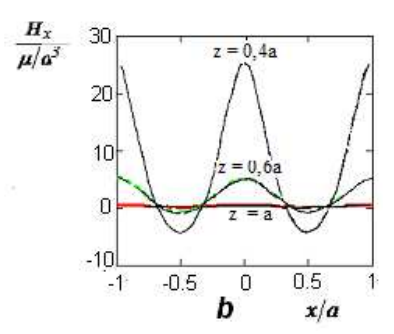

The effective magnetic charge approximation was used [13-17] to calculate the magnetic field distribution. If the ferromagnetic rod radius is small the rod tops can be considered as point-like "magnetic charges" $q_{M}=M_{s} \pi d^{2} / 4$, while the rod bottoms as $-q_{M}$. In this case the magnetic potential takes the following form:

Fig. 3. a) the model structure; $b$ ) the magnetic potential distribution along $x$ axis for different $z$.

$$
\psi(\mathbf{r})=q_{M} \sum_{n=-\infty}^{\infty} \sum_{m=-\infty}^{\infty}\left\{\left((x-a \cdot n)^{2}+(y-a \cdot m)^{2}+z^{2}\right)^{1 / 2}-\left((x-a \cdot n)^{2}+(y-a \cdot m)^{2}+\left(z+\left|z_{0}\right|\right)^{2}\right)^{1 / 2}\right\}
$$

$$
\begin{aligned}
& \text { If } z>0 \\
& \qquad \psi(\mathbf{r})=\sum_{n=-\infty}^{\infty} \sum_{m=-\infty}^{\infty} \frac{z \cdot q_{M} z_{0}}{\left((x-a \cdot n)^{2}+(y-a \cdot m)^{2}+z^{2}\right)^{3 / 2}}
\end{aligned}
$$

Here $q_{M}|z|_{0}$ is the rod magnetic moment $\mu$. Thus there is only one dimensional parameter (the lattice period) in the system. Substituting $\xi=x / a, \eta=y / a, \zeta=z / a$ the formula (2) can be rewritten as:

$$
\psi(\mathbf{r})=\frac{\mu}{a^{2}} \sum_{n=-\infty}^{\infty} \sum_{m=-\infty}^{\infty} \frac{\zeta}{\left((\xi-n)^{2}+(\eta-m)^{2}+\zeta^{2}\right)^{3 / 2}}
$$

This allows to calculate magnetic fields in an arbitrary point in space, with $z>0$. The field is substantially nonuniform and gradient only for the heights lower, than $z \sim a$.
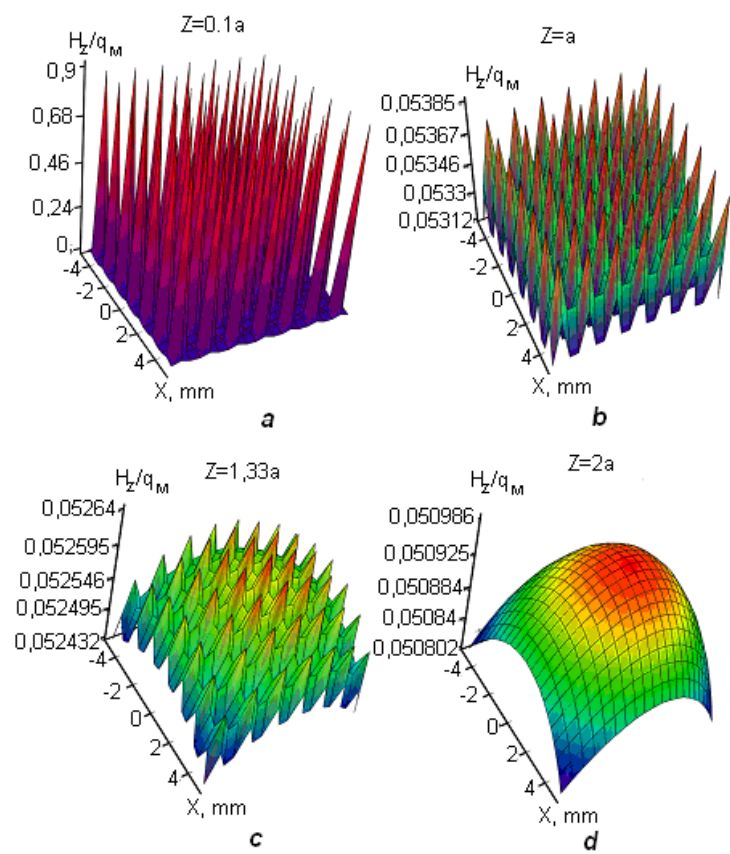

Fig. 4. Magnetic potential distribution as a function of $x$ and $y$ when: $a) z=$ $0.1 a, b) z=a, c) z=1,33 a, d) z=2 a . Z_{0}=20 \mu \mathrm{m}, a=1.5 \mathrm{~mm}$.
The Fig. 4 shows the results of calculation of the magnetic potential distribution above the array using (3) for the different $z$. To increase the gradient field height the magnetization modulation period should be increased, but this leads to the decrease of the field nonuniformity.

\subsubsection{Experimental Dispersion Field Measurements}

To determine the magnetic field distribution the magnetic field intensity space distribution was measured first over the surface of the soft magnetic substrate. The measurements have been performed using the Hall sensor and moving the plate along $x$ direction at different $z$. The magnetic field intensity distribution is presented in Fig.5. It is worth to be noted that the Hall sensor size is about $1.5 \mathrm{~mm}$ which leads to some averaging of the results.
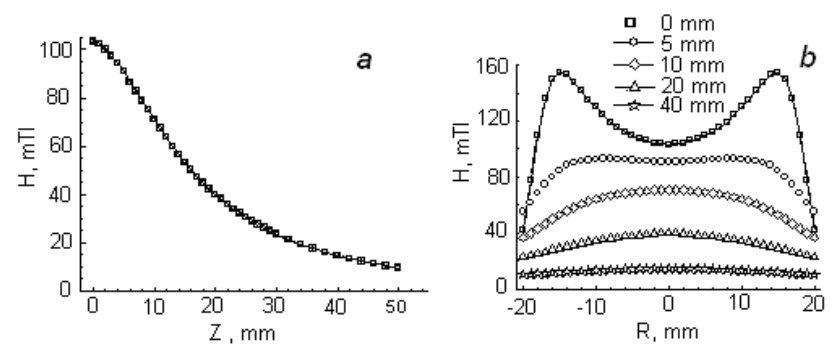

Fig. 5. a) magnetic field distribution as a function of $z$; b) magnetic field distribution along the $x$ direction at different $z$.

The results of the measurements demonstrate of magnetic field inhomogeneity with $z$. But the inhomogeneous fields height is much higher than the theoretical one. The study of the magnetic field over the resin-bonded magnets has been done using set-up with moving Hall sensor allowing scanning in the XY plane and gradual motion along $z$ axis. The results of the measurements are presented in Fig. 6

The results clearly show that the intensity of stray magnetic field decreases with $z$ while its homogeneity increases. 

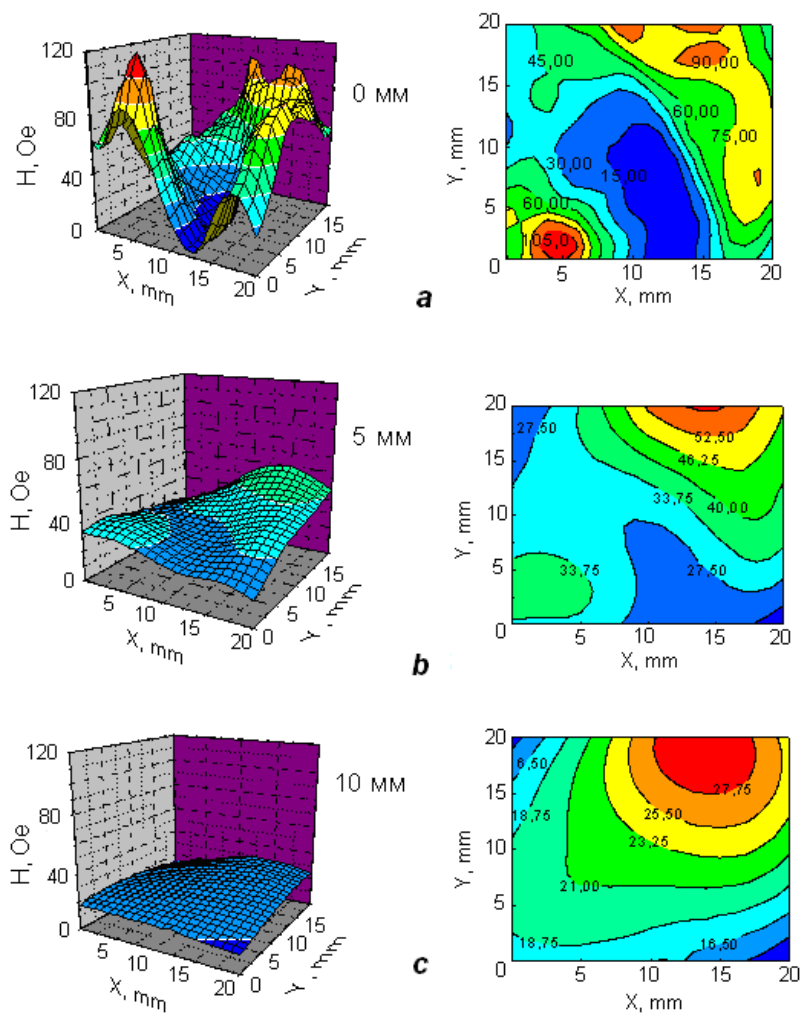

Fig. 6. Magnetic field intensity distribution above the surface of the resinbonded magnets at $\mathrm{z}=0(\mathrm{a}), 5(\mathrm{~b})$ and $10(\mathrm{c}) \mathrm{mm}$.

\section{Results and Discussion}

\subsection{Magnetic Field Influence on Cells Adhesion, Morphology and Proliferation}

As it has been mentioned above there is no general consensus about mechanisms of the influence of moderate magnetic field on biosystems. But there is a supposition that the most sensitive element to the magnetic field action a cell plasmolemma due to its bioelectric properties [18, 19]. It has been reported previously that SMF of medium intensity can change plasmolemma functions in different types of cells [20-25]. One of the most important functional characteristic is its ability to adhesion. Adhesion receptors take part also in cell migration, proliferation and differentiation processes which are the basis for the tissue and organ architectonics [26].

We have studied the effect of intensity and gradient of SMF generated by resin-bonded magnets on the cell adhesion, morphology and proliferation. The ability of the cells to attach themselves to the cultural plastic under the action of the external magnetic field had been studied in our experiments. The scheme of experiments is shown in Fig.7.

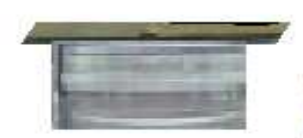

1

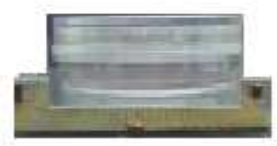

2
Fig. 7. Image the scheme of the experiments.
During the first experiment the Petri dish is situated under the resin-bonded magnets so the cells were well separated from the magnets. The magnetic field gradient was directed the bottom of the Petri dish throughout culture medium. During the second experiment the Petri dish was situated on the magnet. In this case the magnetic field gradient was directed toward the bottom of the dish.

The cells adhesion property was evaluated as a number of cells which were able to attach themselves to the host material in 24 hours. The attachment effectiveness has been calculated according to the formula [27]: $E=[(\mathrm{m}-$ $\mathrm{n}) / \mathrm{m}] \cdot 100 \%$, where $\mathrm{E}$ is the adhesion index; $\mathrm{m}$ is the number of cultured cells; $n$ is the amount of nonattached cells. The diagram of the adhesion index is shown in Fig.8. The experimental data have proved that the cell attachment effectiveness has the highest level when the magnetic film is produced by magnets situated above the Petri dish (the first experiment).

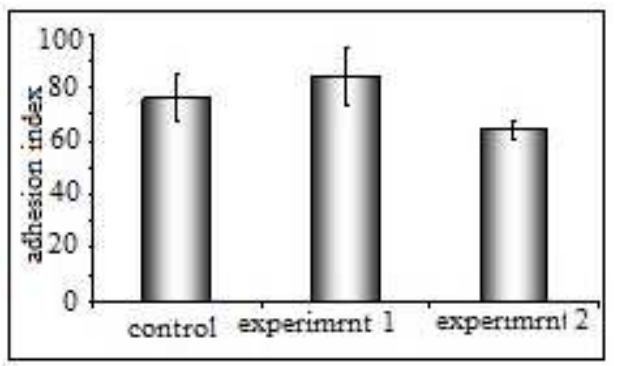

Fig. 8. The cells adhesion index obtained in different experiments with and without magnetic field.

The adhesion index that was obtained in the first experimen was well above one obtained in the control experiment without magnetic field $(84,25 \pm 10,25$ i 76,25 \pm 8,83 correspondingly). The number of attached cells during the second experiment was significantly smaller then during the control one (Fig. 8).

Another important functional characteristic of cells at single-layered cultivation is the proliferation rate. The cells proliferative suspension ability for in vitro cultivation for the different positions of the magnet is presented in Fig .9.

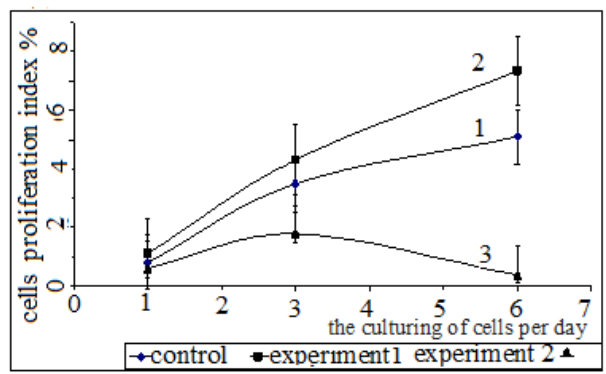

Fig. 9. Time dependence of cells proliferation index.

The behavior of proliferation rate dependences for the first experiment and for the control one is quit similar (Fig. 9, curves 1 and 2). But the cells proliferation index on the sixth cultivation day in the first experiment was $(7,35 \pm 1.2)$ was 
much higher then this index in control experiment $(5,1 \pm$ 0,95). The second experiment (Fig. 9, curve 3) demonstrates slowing of cell proliferation process. The substantial index decrease in comperison with the control experiment was observed on the third cultivation day and the cell growth was totally inhibited on the sixth day of the cultivation.

The cells proliferation ability has been studied at the single-layer cell cultivation and has been determined as the ratio of the number of the cultured cells (K2) to the number of the cultivated cells (K1) on the first, third and sixth day of the experiment ( $\mathrm{K} 1$ and $\mathrm{K} 2$ are the proliferation indexes). In the six day cultivation time there was no confluence detected in the control. The cell number calculation has been conducted in the Goryaev's count chamber using a standard laboratory method. The vital cell morphology and the singlelayer formation rate have been measured on the first, the third and the sixth cultivation day using the Olympus inverted microscope equipped with the Scopetek GSM-130E photocamera.

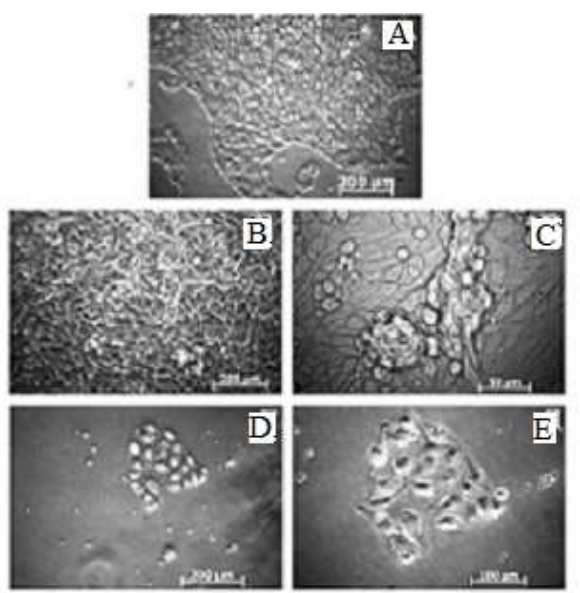

Fig. 10. The cells culture morphology on the sixth cultivation day. $A$ is the control data; $B$ and $C$ are the first experiment results; $D$ and $E$ are the second experiment results.

As we can see the cells single-layer did not reach confluence on the sixth cultivation day (Fig. $10 \mathrm{~A}$ ), while the "overgrowth" of cell culture and the appearance of 3D conglomerates (Fig. 10, B, C) can be observed in the first experiment. The external magnetic field is presumably having a negative effect on cell culture (Fig. 10, D, E) in the second experiment: the formation of single-layer haven't taken place, only smal cell formations consisting of a couple of cells are distinguishable.

It can be concluded that the cells adhesion, morphology and proliferation rate could be determined not only by the magnetic field intensity, but also by the magnetic field gradient direction. The adhesion and the proliferation are much lower in the second experiment and quit higher in the first one in comparison with the control. The cell culture functional characteristics change under the action of SMF. Probably these changes are related to some changes in plasmalemma bioelectric characteristics.

It should be that the proposed magnetic field system can be used to control imbedded into the cell nanoparticles distribution or for targeted drug delivery. We have done some test experiments as to the possibility to control the distribution of nanoparticles in cells using the resin-bonded magnets which charateristics are shown in Fig. 11.
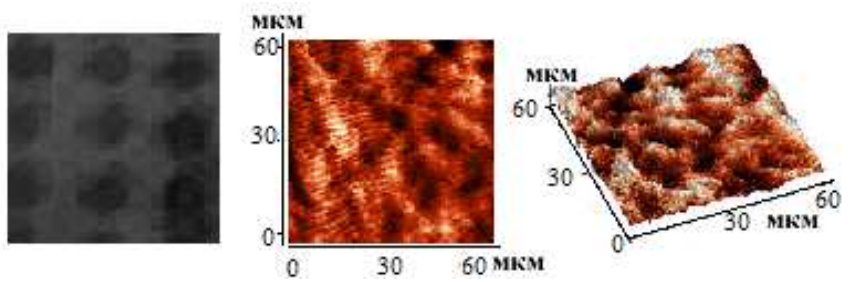

Fig. 11. a) magnetic substrate; b) 2D image of magnetic field lines distribution at $400 \mathrm{~nm}$ distance from c) $3 D$ image of magnetic field lines distribution.

Such a magnetic field configuration favors to the homogeneous nanoparticles distribution in such biological objects during using magnetic particles assisted drug delivery and their localization in the certain area. Homogeneous nanoparticles distribution can play an essential role in oncology for magnetic hypothermia methods. The preliminary data on nanoparticles distribution in cells with and without magnetic substrate (Fig.12) are presented here as an example. [28]

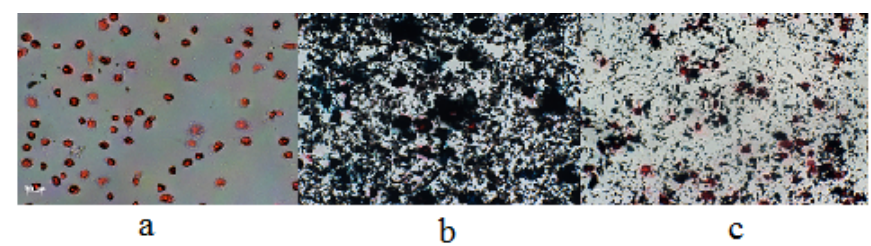

Fig. 12. a) cell culture, b) cell culture with nanoparticles imbedded into it c) cell culture with nanoparticles imbedded into it with magnetic interlayer.

\section{Conclusions}

We have developed the gradient magnetic fields source which can be used in biotechnology.

1. The possibility of magnetic control 3D cell growth without polymer matrixes or magnetic nanoparticles has been shown.

2. Cell adhesion, morphology and proliferation rate could be determined not only by magnetic field intensity but also by the magnetic field gradient direction.

3. The increase and decrease of the proliferation rate with respect to control experiments (without magnetic field) was observed depending on the direction of the magnetic field gradient.

\section{References}

[1] Hubert Tseng, Jacob A. Gage, Robert M. Raphael, Robert H. Moore, Thomas C. Killian, K. Jane Grande-Allen and Glauco R.Souz, "Assembly of a Three-Dimensional Multitype Bronchiole Coculture Model Using Magnetic Levitation" Tissue Engineering: Part C, vol.19, № 9, pp.1-11, 2013. 
[2] T.A. Ignateva, V.N. Voevodin, P.A. Kutsenko, V.V. Kalinovskiy, Y.I. Dzhezherya, V.O. Golub, V.V. Kiroska, “The development of magnetic substrates with determined $3 \mathrm{~d}$ geometry of magnetic field for the biotechnology applications", Paper presented at the International Conference «ImagineNano» nanoBio\&Med, Bilbao Exhibition Center, pp.196-197, 2013. (references)

[3] T.A. Ignateva, V.N. Voevodin, P.A. Kutsenko, V.V. Kalinovskiy, Y.I. Dzhezherya, V.O. Golub, I. Sharai, A.N. Goltsev, V.V. Kiroshka, T.A. Urchyk, "Magnetic substrates with determined $3 \mathrm{~d}$ geometry of magnetic field for the biotechnology applications", Paper presented at the International Conference "Functional Materials "ICFM, Partenit, Ukraine, p.461, 2013. (references)

[4] V.V. Kiroshka, T.A. Urchyk, V.N. Voevodin, T.A. Ignateva, A.M. Bovda, V.O. Golub, "Effect of static magnetic field on proliferation and adhesion of the cell culture", Paper presented at the International Conference "Functional Materials" ICFM, Partenit, Ukraine, p.462, 2013(references)

[5] Hong F.T. "Magnetic field effects on biomolecules, cells, and living organisms BioSystems”, vol.36, №2, pp.187-229, 1995.

[6] A.D. Rosen "Mechanism of action of moderate-intensity static magnetic fields on biological systems", Cell. Biochem. Biophys, vol. 39, №2, pp.163-173,2003.

[7] W.B. High, J.Sikora J, K.Ugurbil, M.Garwood. "Subchronic in vivo effects of a high static magnetic field $(9.4 \mathrm{~T})$ in rats", $\mathrm{J}$. of Magn. Reson. Imaging, vol.12, №2, pp.122-139, 2000.

[8] J.F. Schenck, "Safety of strong, static magnetic fields", J. of Magn. Reson. Imaging, vol.12, №1, pp.2-19, 2000.

[9] J.A.Strand, C.S.Abernethy, J.R.Skalski, R.G.Genoway, "Effects of magnetic field exposure on fertilization success in rainbow trout, Salmo gairdneri”, Bioelectromagnetics, vol.4, №4, pp. 295-301. 1983.

[10] H.B.Brewer, "Some preliminary studies on the effects of a static magnetic field on the life cycle of Lebistes reticulates (guppy),” Biophys. J., vol. 28, №4, pp. 305-314, 1979.

[11] W.B. High, J. Sikora, K.Ugurbil, M.Garwood, "Subchronic in vivo effects of a high static magnetic field $(9.4 \mathrm{~T})$ in rats", J. of Magn. Reson. Imaging, vol.12, №2, pp.122-139, 2000.

[12] V.L.Mironov, "Fundamentals of Scanning Probe Microscopy," Institute of Physics of Microstructures, The Russian Academy of Science, Nizhniy Novgorod, 2004.

[13] L.D. Landau, E.M. Lifshitz, "The Classical Theory of Fields," Course of Theoretical Physics A, vol.2, Pergamon Press, 1971.

[14] C. Kittel "Theory of the structure of ferromagnetic domains in films and small particles," Phys. Rev, vol. 70, №11-12, pp. 965-971, 1946.

[15] V.G. Baryakhtar, Y.I. Gorobets, "Cylindrical magnetic domains and their arrays,” Kiev, Naukova, 168 p. 1988.
[16] Y.I. Dzhezherya, M.V. Sorokin, E.A. Bubuk, "Magnetostatic domain structures," Naukovi visti NTUU KPI, N 4, pp. 51-54, 2006.

[17] Y.I. Dzhezherya, M.V. Sorokin, E.A. Bubuk, "Inhomogeneous configurations of magnetization in ferromagnetic films with fourfold anisotropy," JETP, vol. 133, N4, pp.844-851, 2007.

[18] M. H. Repacholi, B. Greenbaum, "Interaction of static and extremely low frequency electric and magnetic fields with living systems: health effects and research needs," Bioelectronmagnetics, vol. 20, №4, pp.133-160, 1999.

[19] L. Teodori, W. Gohde, M. GValente., F. Tagliaferri, D.Colett, "Static magnetic fields affect calcium fluxes and inhibit stressinduced apoptosis in human glioblastoma cells," Cytometry, vol. 49, №4, pp.143-149, 2002.

[20] A. Chionna, M. Dwikat, E. Panzarini, B. Tenuzzo, "Cell shape and plasma membrane alterations after static magnetic fields exposure,” Eur J Histochem, vol. 47, №4, pp.299-308, 2003.

[21] S.H. Hamada, R. Witkus, Jr.R. Griffith, "Cell surface changes during electromagnetic field exposure," Exp. Cell Biol, vol. 57, №1, pp.1-10, 1989.

[22] A.Lisi, D.Pozzi, E.Pasquali, S. Rieti, "Three dimensional (3D) analysis of the morphological changes induced by $50 \mathrm{~Hz}$ magnetic field exposure on human lymphoblastoid cells (Raji)," Bioelectromagnetics, vol. 21, №, pp. 46-5, 2000.

[23] S. Paradisi, G. Donelli, M.S. Santini, E..Straface W. A. Malorni, "50 Hz magnetic field induces structural and biophysical changes in membranes," Bioelectronmagnetics. vol. 14, №3, pp.247-255, 1993.

[24] S.Rieti, V.Manni, A.Lisi, L.Giuliani, "SNOM and AFM microscopy techniques to study the effect of non-ionizing radiation on the morphological and biochemical properties of human keratinocytes cell line (HaCaT),'J. of Micros, vol. 213, №1, pp.20-28, 2004.

[25] N.Santoro, A.Lisi, D.Pozzi E.Pasquali, A.Serafino, "Effect of extremely low frequency (ELF) magnetic field exposure on morphological and biophysical properties of human lymphoid cell line (Raji)," Bioch. Biophys. Acta, vol.1357, №3, pp..281-290, 1997.

[26] K.Bhadriraju, L.K.Hansen, "Extracellular matrix and cytoskeleton-dependent changes in cell shape and stiffness," Exp. Cell. Res. vol.278, №1, pp.92-100, 2002.

[27] J. M. Davis, "Basic cell culture," Second ed. Oxford: Univ. Press, pp.381, 2001.

[28] Voyevodin V.N., Ignatyeva T.A., Bovda A.M., P.A. Kutsenko, V.O.Golub, I.V.Sharay, V.V. Kiroshka, "Magnetic matrix with 3D magnetic field configuration," Nanostructure Materials 2012: Russia-Ukraine-Belorus»,-Abstract Book, pp.274, 2012. 